\title{
Barreiras e facilitadores para a atividade física em pessoas com déficit intelectual
}

\section{Barriers and facilitators to physical activity people with intellectual disability}

\author{
Laura Garcia Jung'; Angélica Xavier Kalinoski'; Alexandre Carriconde Marques
}

\section{Resumo}

O objetivo deste estudo foi descrever as barreiras e os facilitadores da prática de atividade física $(\mathrm{AF})$ no tempo livre e sua associação com essa prática em pessoas maiores de seis anos de ambos os sexos com déficit intelectual (DI) inseridas nas Associações de Pais e Amigos dos Excepcionais (APAES). A amostra foi composta por 1.191 pessoas com DI representadas pelos seus responsáveis. As barreiras e facilitadores para a prática de AF foram identificadas com questões fechadas em que o responsável respondia se o fator influenciava sempre (1), influenciava às vezes (2) ou não influenciava (3) na prática de AF. As questões foram separadas em três dimensões que correspondiam a onze fatores de ordem pessoal, sete de ordem ambiental e dez de ordem social. Verificou-se no aspecto pessoal sete facilitadores e duas barreiras, nos quais destaca-se o fato de sentir-se capaz como facilitador associado com a prática de AF e não possuir dinheiro como barreira; no aspecto ambiental seis facilitadores e uma barreira, sendo a companhia de pessoas próximas o principal facilitador pela associação com a prática de AF e a ausência de projetos sociais como barreira; e no aspecto social cinco facilitadores e uma barreira, no qual encontram-se a existência de locais próximos de casa e os profissionais estarem preparados para trabalhar com pessoas com DI os principais facilitadores e a falta de companhia como barreira. Conclui-se que as barreiras e facilitadores de AF para as pessoas com DI podem estar ligados ao estímulo que recebem, pois a companhia de outra pessoa foi o principal facilitador associado com a prática, isto pode ocorrer devido às limitações que a maioria possui no aspecto social. Oferta de locais com qualidade para prática de AF também facilitam a prática de $\mathrm{AF}$, o que pode ser amenizado com o investimento em políticas públicas e projetos sociais de qualidade.

\section{Palavras-chave}

Déficit Intelectual; Barreiras e facilitadores; Atividade física; Tempo livre.

\begin{abstract}
The objective of this study was to describe the barriers and facilitators of free time physical activity (PA), their influence both in this practice of people with intellectual deficit (ID) inserted in APAES / RS. The sample consisted of 1191 ID individuals represented by their legal representatives. The barriers and facilitators for the practice of PA were identified with closed questions in which the respondent answered whether the factor always influences (1), sometimes influences (2) or does not influence (3) the practice of PA. The issues were separated into three dimensions: personal, environmental and social. As a result, seven facilitators and two barriers were observed in the personal aspect, in which the fact that they feel capable as a facilitator associated with the practice of $P A$ and lack of money as a barrier; In the environmental aspect, six facilitators and a barrier, the company of close people being the main facilitator by the association with the practice of PA and non-existence of social projects as a barrier; And in the social aspect five facilitators and a barrier in which the existence of places close to their homes and professionals prepared to work with people with ID appear as the main facilitators and lack of companion as a barrier. It is concluded that some barriers and facilitators are linked to the stimulus that the people receive, since they can arouse the taste for $P A$ if there is company, providing conditions for the practice due to the limitations that the majority has in the social aspect. Offering quality $P A$ sites also facilitate, which can be provided by the investment in public policies and social projects.
\end{abstract}

\section{Keywords}

Intellectual disability; Barriers and facilitators; Physical activity; Leisure time.

\section{Introdução}

A expectativa de vida da população tem aumentado ao longo das últimas décadas ${ }^{1}$ e, ao mesmo tempo, a tecnologia traz facilidades para as atividades cotidianas, o que as tornam menos desgastantes fisicamente e com menor dispêndio de energia corporal. Tais con- 
dições têm determinado, de forma predominante, comportamentos sedentários que desencadeiam o aumento das doenças crônicas não transmissíveis (DCNTs), as quais podem ser agravadas nas pessoas com deficiências ${ }^{3,4}$.

Essa parte da população possui necessidades de cuidado à saúde adicionais e mais complexos do que os da população em geral ${ }^{5,6}$. Possuem também limitações para a realização de atividades cotidianas associadas às deficiências. Estudos mostram que o nível de AF das pessoas com DI é inferior ao da população em geral ${ }^{7-9}$, fato preocupante, tendo em vista que a prática de atividades físicas são importantes na prevenção das DCNTs ${ }^{10}$.

Para a prática de AF, fatores caracterizados como barreiras ou facilitadores podem ser determinantes na aderência ou não aos programas de $\mathrm{AF}^{11}$. Percebe-se que mais relevante do que a informação sobre a importância da prática de AF, são as oportunidades dadas às pessoas. Sendo assim, se elas forem impedidas de serem ativas devido às barreiras originadas pela própria deficiência, principalmente o preconceito, essas informações não representariam mudanças no estilo de vida ${ }^{12}$.

Há na literatura brasileira um estudo sobre barreiras e facilitadores para pessoas com deficiência motora, sendo apontados o apoio dos amigos e família, a simpatia e conhecimento do profissional como principais facilitadores e as limitações físicas e a falta de programas específicos como as principais barreiras ${ }^{13}$. No entanto, especificamente para as pessoas com DI há carência na literatura brasileira sobre a identificação das barreiras e facilitadores para prática de $\mathrm{AF}$, mesmo sendo um fator importante para a qualidade de vida ${ }^{14}$.

O conhecimento dessas barreiras e facilitadores da prática de AF, torna-se importante para propiciar o aumento das oportunidades para as pessoas com DI terem um estilo de vida ativo. Em razão disso, o objetivo do presente trabalho foi descrever as barreiras e os facilitadores da prática de AF no tempo livre, sua associação com essa prática das pessoas com DI maiores de seis anos inseridas nas APAES do Rio Grande do Sul.

\section{Métodos}

O presente estudo é caracterizado como epidemiológico transversal e teve como população alvo pessoas com DI, maiores de seis anos, que frequentam as APAEs no estado do Rio Grande do Sul. A seleção da amostra foi não probabilística intencional, pois oportunizou a participação de todas as pessoas que frequentam as APAEs no estado do Rio Grande do Sul.

A coleta de dados foi realizada no ano de 2012 e partiu de um contato inicial com a Federação das APAEs do RS (FEAPAES-RS). A presidente autorizou o estudo disponibilizando uma carta de anuência e em seguida foram contatados os conselhos que estão distribuídos pelo estado. Os conselheiros ficaram responsáveis por enviar uma relação com o número de alunos maiores de seis anos matriculados em sua entidade.

Partindo das listas recebidas, foram enviados pelo correio a quantidade necessária de questionários para cada uma das APAEs. No total, foram distribuídos 5000 questionários. As instituições encaminharam aos pais e responsáveis o questionário, fornecendo todas as instruções para o seu preenchimento, bem como o termo de consentimento livre e esclarecido e retornando para o responsável pelo estudo, via correios.

O questionário utilizado na coleta de dados foi construído e adaptado com instrumentos já validados para avaliação do perfil do estilo de vida ${ }^{12}$, composto por questões referentes a dados de identificação (data de nascimento, estatura, 
massa corporal e sexo)); quantidade de bens, sendo que a condição financeira foi estimada através do poder de compra e as pessoas foram categorizadas em quatro níveis de acordo com suas condições)prática de AF no tempo livre estimada pelo tempo diário e frequência semanal de cada prática (minutos por semana).$^{15}$.

As barreiras e facilitadores para a prática de AF foram identificadas com questões fechadas em que o responsável respondia se o fator influenciava sempre (1), influencia às vezes (2) ou não influencia (3) na prática de AF em cada uma das perguntas. As questões foram separadas em três dimensões: pessoal, ambiental e social ${ }^{15}$, conforme segue:

- Aspecto pessoal: Ele gosta e tem prazer em praticar AF? Se sente capaz de praticar AF? Possui dinheiro para praticar AF que tenha algum custo? A timidez o impede de praticar AF? Sente preguiça de praticar AF? Possui experiências negativas em relação à prática de $\mathrm{AF}$ ? Prefere outras atividades ao invés de $\mathrm{AF}$ ? Tem medo de se machucar praticando atividade física? Possui tempo livre para praticar AF? Possui alguma doença que o impeça de praticar atividade física? Possui limitações físicas/ motoras para a prática de AF?

- Aspecto ambiental: Há locais disponíveis próximos de casa para a prática de AF? Se existem locais disponíveis, eles são acessíveis para a prática? A comunidade o acolhe de forma adequada? Os profissionais de educação física são preparados para atendê-lo? Existem projetos sociais de AF próximos a sua casa?

- Aspecto social: A família acha importante a prática de AF? Os pais acham a AF importante para manter a aparência? Os pais acham a AF importante para a saúde? Os pais possuem tempo para acompanhar o filho em atividades físicas? Os pais ficam constrangidos com o filho praticando AF? Alguém que mora na mesma casa pratica AF? Possui companhia dos parentes para praticar AF? Possui companhia de amigos para praticar AF? Tem apoio das pessoas próximas para praticar $\mathrm{AF}$ ? Algum profissional já recomendou a prática de $\mathrm{AF}$ ?

\section{Análise de dados}

As percepções sobre barreiras e facilitadores foram determinadas de acordo com a prevalência das respostas e o teor da pergunta, se a caracterizava como fator que facilitava ou que dificultava a prática de AF. Já os que responderam que o fator influenciava "às vezes", foram unidos ao fator prevalente também de acordo com o teor da pergunta, se era facilitador ou barreira.

$\mathrm{Na}$ análise de dados foram empregados recursos da estatística descritiva para os dados demográficos: distribuição de frequências, média, desvio padrão, amplitude. Para verificar as associações entre as variáveis categóricas (prática de AF associada com presença de barreiras e facilitadores por faixa etária AF associada com sexo por faixa etária) foi utilizado o teste do Qui-quadrado. O nível de significância adotado foi de 5\%. Na nálise dos dados foi utilizado o programa Statistical Package for the Social Sciences (SPSS Statistics) 17.0.

\section{Resultados}

A amostra foi composta por 1.191 pessoas com DI representadas no estudo pelos seus responsáveisObservou-se que a maioria das pessoas eram do sexo masculino (55,3\%), com idades entre 11 e 19 anos (45,2\%), e poder de compra das famílias simétrico em relação aos quartis. Verificou-se que 55,5\% $(\mathrm{n}=649)$ praticava AF no lazer, a caminha é a atividade mais praticada com $52,7 \%(\mathrm{n}=342)$ seguido por, andar de bicicleta $35,6 \%(\mathrm{n}=231)$, futebol $31,3 \%(\mathrm{n}=203)$ e dança $24,3 \%(\mathrm{n}=158)$, 
sendo que alguns praticam mais de uma atividade.

Identificou-se que 65,1\% dos meninos, na faixa etária entre 11 e 19 anos, são os que mais praticavam AF no momento livre, porém, essa análise pode ter sido interferida pelo tamanho da amostra em cada faixa etária. Verificaram-se associações estatísticas significativas quando comparado AF X sexo, conforme mostra a

TABELA 1 - Prática de AF no tempo livre das pessoas com Dl por sexo e idade.

\begin{tabular}{lcccccc}
\hline \multirow{2}{*}{ Faixa etária } & \multicolumn{2}{c}{ Homens } & \multicolumn{2}{c}{ Mulheres } & Total & \multirow{2}{*}{ * $^{*}$} \\
\cline { 2 - 5 } & \multicolumn{1}{c}{$\operatorname{Sim}(\%)$} & Não $(\%)$ & $\operatorname{Sim}(\%)$ & Não $(\%)$ & & 0,781 \\
\hline $07-10$ & $79(65,3)$ & $42(34,7)$ & $36(63,2)$ & $21(36,8)$ & 178 & 0,007 \\
$11-19$ & $194(65,1)$ & $104(34,9)$ & $126(53,6)$ & $109(46,4)$ & 534 & 0,859 \\
$20-30$ & $71(47,7)$ & $81(52,3)$ & $56(46,7)$ & $64(53,3)$ & 276 & 0 \\
$31-40$ & $23(46,9)$ & $26(53,1)$ & $24(40,7)$ & $35(59,3)$ & 108 & 0,514 \\
$>40$ & $15(46,9)$ & $17(53,1)$ & $21(39,6)$ & $32(60,4)$ & 85 & 0,512 \\
Total & $382(58,6)$ & $270(41,4)$ & $263(50,2)$ & $261(49,8)$ & 1181 & 0,003 \\
\hline
\end{tabular}

*Teste Qui-quadrado.

A tabela 2 apresenta informações sobre os facilitadores e ou barreiras mais prevalentes.

TABELA 2 - Percepção dos responsáveis sobre as barreiras e facilitadores da prática de AF no tempo livre das pessoas com DI.

\begin{tabular}{|c|c|c|c|c|c|c|}
\hline \multirow{2}{*}{ Barreiras / Facilitadores } & \multirow[b]{2}{*}{ Todos (\%) } & \multicolumn{5}{|c|}{ Faixa etária (anos) } \\
\hline & & $7-10$ & $11-19$ & $20-30$ & $30-40$ & $>40$ \\
\hline \multicolumn{7}{|l|}{ PESSOAIS } \\
\hline Gosta e tem prazer em realizar AF & $F(90,4)$ & $\mathrm{F}$ & $\mathrm{F}$ & $\mathrm{F}$ & $\mathrm{F}$ & $\mathrm{F}$ \\
\hline É capaz de praticar AF & $F(91,3)$ & $\mathrm{F}$ & $\mathrm{F}$ & $\mathrm{F}$ & $\mathrm{F}$ & B \\
\hline Não possui dinheiro para praticar AF & $B(77,0)$ & B & B & B & B & B \\
\hline Não é tímido para praticar AF & $F(60,6)$ & $\mathrm{F}$ & $\mathrm{F}$ & $\mathrm{F}$ & $\mathrm{F}$ & $\mathrm{F}$ \\
\hline Não sente preguiça de praticar AF & $F(55,3)$ & $\mathrm{F}$ & $\mathrm{F}$ & $\mathrm{F}$ & $\mathrm{F}$ & $\mathrm{F}$ \\
\hline Não possui experiências negativas com AFs & $F(78,3)$ & $\mathrm{F}$ & $\mathrm{F}$ & $\mathrm{F}$ & $\mathrm{F}$ & $\mathrm{F}$ \\
\hline Prefere outras atividades & $B(57,2)$ & $\mathrm{F}$ & B & B & B & B \\
\hline Não tem medo de machucar praticando AF & $F(54,0)$ & $\mathrm{F}$ & $\mathrm{F}$ & $\mathrm{F}$ & $\mathrm{F}$ & $\mathrm{F}$ \\
\hline Possui tempo livre para praticar AF & $F(92,5)$ & $\mathrm{F}$ & $\mathrm{F}$ & $\mathrm{F}$ & $\mathrm{F}$ & $\mathrm{F}$ \\
\hline \multicolumn{7}{|l|}{ AMBIENTAIS } \\
\hline Há locais disponíveis próximos de casa & $F(51,2)$ & $\mathrm{F}$ & $\mathrm{F}$ & B & B & $\mathrm{F}$ \\
\hline Os locais são acessíveis & $F 59,7)$ & $\mathrm{F}$ & $\mathrm{F}$ & $\mathrm{F}$ & B & $\mathrm{F}$ \\
\hline A comunidade o acolhe de forma adequada & $F(83,3)$ & $\mathrm{F}$ & $\mathrm{F}$ & $\mathrm{F}$ & $\mathrm{F}$ & $\mathrm{F}$ \\
\hline Os profissionais são preparados & $F(80,3)$ & $\mathrm{F}$ & $\mathrm{F}$ & $\mathrm{F}$ & $\mathrm{F}$ & $\mathrm{F}$ \\
\hline Não há projetos sociais de AF próximos de casa & $B(80,4)$ & B & $\mathrm{B}$ & B & B & B \\
\hline O clima da região favorece a prática de AF & $F(89,3)$ & $\mathrm{F}$ & $\mathrm{F}$ & $\mathrm{F}$ & $\mathrm{F}$ & $\mathrm{F}$ \\
\hline O ambiente ao redor de casa é seguro & $F(86,0)$ & $\mathrm{F}$ & $\mathrm{F}$ & $\mathrm{F}$ & $\mathrm{F}$ & $\mathrm{F}$ \\
\hline \multicolumn{7}{|l|}{ SOCIAIS } \\
\hline A família acha importante a prática de AF & $F(99,0)$ & $\mathrm{F}$ & $\mathrm{F}$ & $\mathrm{F}$ & $\mathrm{F}$ & $\mathrm{F}$ \\
\hline Os pais possuem tempo para acompanhar o filho em Afs & $F(82,0)$ & $\mathrm{F}$ & $\mathrm{F}$ & $\mathrm{F}$ & $\mathrm{F}$ & $\mathrm{F}$ \\
\hline Os pais ficam constrangidos com o filho praticando AF & $F(91,2)$ & $\mathrm{F}$ & $\mathrm{F}$ & $\mathrm{F}$ & $\mathrm{F}$ & $\mathrm{F}$ \\
\hline Alguém que mora na mesma casa pratica & $F(61,9)$ & $\mathrm{F}$ & $\mathrm{F}$ & $\mathrm{F}$ & $\mathrm{F}$ & $\mathrm{F}$ \\
\hline Possui companhia de parentes e amigos & $B(63,4)$ & $\mathrm{F}$ & $\mathrm{B}$ & B & $\mathrm{F}$ & B \\
\hline Tem apoio das pessoas próximas & $F(78,5)$ & $\mathrm{F}$ & $\mathrm{F}$ & $\mathrm{F}$ & $\mathrm{F}$ & $\mathrm{F}$ \\
\hline
\end{tabular}

$\mathrm{B}=$ Barreira para a prática de AF; F= Facilitador para a prática de AF. 
Nos aspectos pessoais, observa-se que as principais barreiras para a prática de AF foram: a falta de dinheiro e a preferência por outras atividades, entretanto, nas crianças é possível notar a preferência por AF em relação a outras e isso aponta um facilitador. No grupo acima de 40 anos, percebe-se que o fato de não se sentirem capazes de praticar AF é uma barreira, bem como possuírem menos tempo livre para a prática.

Com relação aos aspectos ambientais, nota-se que a principal barreira é a ausência de projetos sociais próximos de casa. Já as pessoas entre 20 e 40 anos relataram que a falta de locais para a prática de $\mathrm{AF}$ também é um fator que dificulta a prática de AF. Para os responsáveis pelas pessoas entre 20 e 30, que percebem a presença de locais disponíveis próximos de casa, destacam que eles não são acessíveis.

Quanto aos aspectos sociais, a falta de companhia é o principal fator identificado como barreira para a prática de $\mathrm{AF}$, a não ser para as pessoas com idades entre 07 e 10 anos, bem como para os que têm entre 30 e 40 anos que não possuem barreiras neste aspecto.

Ao relacionar os aspectos pessoais definidos como barreiras ou facilitadores com a prática de $\mathrm{AF}$, observou-se uma associação significativa entre o facilitador gostar e praticar AF nos homens com idades de 07-10 anos e acima de 40 anos ( $\mathrm{p}<0,05)$. Houve também uma associação significativa entre o facilitador sentir-se capaz de praticar AF e ser homem maior de 10 anos e ser menina maior de 20 anos ( $\mathrm{p}<0,05)$. A barreira de falta de dinheiro para praticar AF foi significativo com o fato de não praticar AF no grupo de mulheres com idades de 31 a 40 anos ( $p<0,05)$. Não possuir timidez está associado como facilitador para a prática de AF nas mulheres de 07 a 10 anos e acima de 40, bem como nos homens de 31 a 40 anos. Não ter experiência negativa está associado como facilitador para a prática de AF nas mulheres entre 20 e 30 anos. Não preferir outras atividades está associado como facilitador com a prática de $\mathrm{AF}$ nos homens entre 31 e 40 anos. O facilitador de ter tempo livre está associado com a prática nas mulheres acima de 40 anos. As barreiras de ter preguiça ou medo de se machucar não apresentaram associações

Nos aspectos ambientais, o facilitador de haver locais disponíveis para a prática está associado com a prática de AF, todavia, para as pessoas entre 20 e 40 anos que percebiam falta de locais disponíveis como uma barreira, não houve associação com a prática de AF, sugerindo assim que a falta de locais é barreira para a prática. O facilitador dos locais disponíveis serem acessíveis está associado com a prática de $\mathrm{AF}$ para as mulheres a partir de 40 anos. A comunidade acolher as pessoas com DI sendo um aspecto facilitador está associado com a prática para as mulheres entre 11 e 19 anos. Os profissionais serem preparados para atendê-los apontados como facilitadores, está associado com a prática nas mulheres nas idades entre 11 e 19 anos e a partir dos 40 anos. O ambiente ao redor de casa ser seguro é um facilitador que está associado com a prática nas mulheres entre 31 e 40 anos, conforme a tabela 4.

Quanto aos aspectos sociais, os pais acharem importante a prática de AF, ter tempo para acompanhar os filhos ou ter apoio de pessoas próximas, apontados como facilitadores, não interferem com associação significativa na prática de AF. O fato de os pais não ficarem constrangidos com os filhos, sendo considerado facilitador, está associado nas mulheres entre 11 e 19 anos e nas de 31 a 40 . Algum familiar praticar AF na mesma casa é um facilitador e foi associado com a prática de AF nas meninas entre 10 e 19 anos. Possuir companhia de parentes ou amigos para praticar como facilitador está associado com a prática de $\mathrm{AF}$ entre as pessoas de 07 a 10 anos e nas mulheres entre 31 e 40 anos, conforme mostra a tabela 5. 
TABELA 3 - Associação entre prática de AF e percepção de cada aspecto pessoal como facilitador por sexo e faixa etária.

\begin{tabular}{|c|c|c|c|c|c|c|c|c|c|c|}
\hline \multirow{4}{*}{ Aspectos Pessoais } & \multicolumn{10}{|c|}{ Faixa etária } \\
\hline & \multicolumn{2}{|c|}{ 07-10 anos } & \multicolumn{2}{|c|}{ 11-19 anos } & \multicolumn{2}{|c|}{ 20-30 anos } & \multicolumn{2}{|c|}{$31-40$ anos } & \multicolumn{2}{|c|}{$>40$ anos } \\
\hline & Masc & Fem & Masc & Fem & Masc & Fem & Masc & Fem & Masc & Fem \\
\hline & $\begin{array}{l}n(\%) \\
p^{*}\end{array}$ & $\begin{array}{l}n(\%) \\
p^{*}\end{array}$ & $\begin{array}{l}n(\%) \\
p^{*}\end{array}$ & $\begin{array}{l}n(\%) \\
p^{*}\end{array}$ & $\begin{array}{c}n(\%) \\
p^{*}\end{array}$ & $\begin{array}{c}n(\%) \\
p^{*}\end{array}$ & $\begin{array}{c}n(\%) \\
p^{*}\end{array}$ & $\begin{array}{c}n(\%) \\
p^{*}\end{array}$ & $\begin{array}{l}\mathrm{n}(\%) \\
\mathrm{p}^{*}\end{array}$ & $\begin{array}{l}\mathrm{n}(\%) \\
\mathrm{p}^{*}\end{array}$ \\
\hline Gosta de AF & $\begin{array}{c}77(68,1) \\
0,001\end{array}$ & $\begin{array}{c}34(65,6) \\
0,261\end{array}$ & $\begin{array}{c}184(66,4) \\
0,156\end{array}$ & $\begin{array}{c}121(54,8) \\
0,166\end{array}$ & $\begin{array}{c}67(48,9) \\
0,424\end{array}$ & $\begin{array}{c}50(48,1) \\
0,430\end{array}$ & $\begin{array}{c}18(42,9) \\
0,274\end{array}$ & $\begin{array}{c}17(35,4) \\
0,086\end{array}$ & $\begin{array}{c}14(60,9) \\
0,011\end{array}$ & $\begin{array}{c}18(41,9) \\
0,635\end{array}$ \\
\hline $\begin{array}{l}\text { É capaz de praticar } \\
\text { AF }\end{array}$ & $\begin{array}{c}74(64,3) \\
0,473\end{array}$ & $\begin{array}{c}34(61,8) \\
0,272\end{array}$ & $\begin{array}{c}169(62,8) \\
0,020\end{array}$ & $\begin{array}{c}122(54,2) \\
0,218\end{array}$ & $\begin{array}{c}75(51,7) \\
0,001\end{array}$ & $\begin{array}{c}56(50,0) \\
0,006\end{array}$ & $\begin{array}{c}23(54,8) \\
0,12\end{array}$ & $\begin{array}{c}24(49,0) \\
0,006\end{array}$ & $\begin{array}{c}15(83,3) \\
0,000\end{array}$ & $\begin{array}{c}21956,8) \\
0,000\end{array}$ \\
\hline $\begin{array}{l}\text { Não possui dinheiro } \\
\text { para AF }\end{array}$ & $\begin{array}{c}12(70,6) \\
0,618\end{array}$ & $\begin{array}{c}5(62,5) \\
0,961\end{array}$ & $\begin{array}{c}41(58,6) \\
0,192\end{array}$ & $\begin{array}{c}27(45,8) \\
0,209\end{array}$ & $\begin{array}{c}13(39,4) \\
0,337\end{array}$ & $\begin{array}{c}12(44,4) \\
0,919\end{array}$ & $\begin{array}{c}2(22,2) \\
0,116\end{array}$ & $\begin{array}{l}1(7,7) \\
0,005\end{array}$ & $\begin{array}{c}3(60,0) \\
0,522\end{array}$ & $\begin{array}{c}3(21,4) \\
0,099\end{array}$ \\
\hline $\begin{array}{l}\text { Não ser tímido para } \\
\text { praticar AF }\end{array}$ & $\begin{array}{c}28(59,6) \\
0,344\end{array}$ & $\begin{array}{c}13(48,1) \\
0,019\end{array}$ & $\begin{array}{c}71(60,7) \\
0,224\end{array}$ & $\begin{array}{c}53(58,2) \\
0,337\end{array}$ & $\begin{array}{c}33(55,9) \\
0,209\end{array}$ & $\begin{array}{c}18(45,0) \\
0,754\end{array}$ & $\begin{array}{c}13(72,2) \\
0,004\end{array}$ & $\begin{array}{c}10(38,5) \\
0,610\end{array}$ & $\begin{array}{c}5(45,5) \\
0,907\end{array}$ & $\begin{array}{c}11(61,1) \\
0,014\end{array}$ \\
\hline $\begin{array}{l}\text { Não sente preguiça } \\
\text { de praticar AF }\end{array}$ & $\begin{array}{c}33(56,9) \\
0,082\end{array}$ & $\begin{array}{c}19(57,6) \\
0,132\end{array}$ & $\begin{array}{c}90(60,8) \\
0,263\end{array}$ & $\begin{array}{c}65(50,0) \\
0,126\end{array}$ & $\begin{array}{c}41(48,2) \\
0,892\end{array}$ & $\begin{array}{c}34(47,9) \\
0,551\end{array}$ & $\begin{array}{c}13(50,0) \\
0,626\end{array}$ & $\begin{array}{c}14(41,2) \\
0,778\end{array}$ & $\begin{array}{c}8(40,0) \\
0,314\end{array}$ & $\begin{array}{c}13(40,6) \\
0,964\end{array}$ \\
\hline $\begin{array}{l}\text { Não ter experiências } \\
\text { negativas com AF }\end{array}$ & $\begin{array}{c}10(71,4) \\
0,839\end{array}$ & $\begin{array}{c}12(63,2) \\
0,957\end{array}$ & $\begin{array}{c}37(67,3) \\
0,655\end{array}$ & $\begin{array}{c}29(54,7) \\
0,880\end{array}$ & $\begin{array}{c}18(54,5) \\
0,278\end{array}$ & $\begin{array}{c}7(26,9) \\
0,020\end{array}$ & $\begin{array}{c}4(66,7) \\
0,261\end{array}$ & $\begin{array}{c}7(50,0) \\
0,397\end{array}$ & $\begin{array}{c}4940,0) \\
0,519\end{array}$ & $\begin{array}{c}5(38,5) \\
0,923\end{array}$ \\
\hline $\begin{array}{l}\text { Não prefere outras } \\
\text { atividades }\end{array}$ & $\begin{array}{c}51(73,9) \\
0,019\end{array}$ & $\begin{array}{c}19(70,4) \\
0,308\end{array}$ & $\begin{array}{c}69(64,5) \\
0,706\end{array}$ & $\begin{array}{c}47(52,8) \\
0,702\end{array}$ & $\begin{array}{c}25(41,0) \\
0,279\end{array}$ & $\begin{array}{c}23(46,9) \\
0,943\end{array}$ & $\begin{array}{c}13(72,2) \\
0,011\end{array}$ & $\begin{array}{c}13(50,0) \\
0,206\end{array}$ & $\begin{array}{c}6(40,0) \\
0,464\end{array}$ & $\begin{array}{c}9(37,5) \\
0,813\end{array}$ \\
\hline $\begin{array}{l}\text { Não ter medo de } \\
\text { machucar na AF }\end{array}$ & $\begin{array}{c}24(54,5) \\
0,105\end{array}$ & $\begin{array}{c}14(56,0) \\
0,245\end{array}$ & $\begin{array}{c}74(60,2) \\
0,115\end{array}$ & $\begin{array}{c}53(58,9) \\
0,227\end{array}$ & $\begin{array}{c}46(52,9) \\
0,172\end{array}$ & $\begin{array}{c}24(47,1) \\
0,863\end{array}$ & $\begin{array}{c}13(48,1) \\
0,831\end{array}$ & $\begin{array}{c}15(42,9) \\
0,778\end{array}$ & $\begin{array}{c}5(33,3) \\
0,200\end{array}$ & $\begin{array}{c}12(40,0) \\
0,939\end{array}$ \\
\hline $\begin{array}{l}\text { Tempo livre para } \\
\text { praticar AF }\end{array}$ & $\begin{array}{c}67(62,6) \\
0,113\end{array}$ & $\begin{array}{c}27(58,7) \\
0,243\end{array}$ & $\begin{array}{c}174(65,4) \\
0,569\end{array}$ & $\begin{array}{c}117(52,2) \\
0,265\end{array}$ & $\begin{array}{c}69(48,6) \\
0,599\end{array}$ & $\begin{array}{c}52(47,3) \\
0,820\end{array}$ & $\begin{array}{c}18(46,2) \\
0,795\end{array}$ & $\begin{array}{c}22(40,7) \\
0,794\end{array}$ & $\begin{array}{c}14(48,3) \\
0,185\end{array}$ & $\begin{array}{c}15(34,9) \\
0,024\end{array}$ \\
\hline
\end{tabular}

*Teste Qui-quadrado.

TABELA 4 - Associação entre prática de AF e percepção de cada aspecto social como facilitador por sexo e faixa etária.

\begin{tabular}{|c|c|c|c|c|c|c|c|c|c|c|}
\hline \multirow{4}{*}{$\begin{array}{l}\text { Aspectos } \\
\text { Sociais }\end{array}$} & \multicolumn{10}{|c|}{ Faixa etária } \\
\hline & \multicolumn{2}{|c|}{ 07-10 anos } & \multicolumn{2}{|c|}{ 11-19 anos } & \multicolumn{2}{|c|}{ 20-30 anos } & \multicolumn{2}{|c|}{$31-40$ anos } & \multicolumn{2}{|c|}{$>40$ anos } \\
\hline & Masc & Fem & Masc & Fem & Masc & Fem & Masc & Fem & Masc & Fem \\
\hline & $\begin{array}{l}n(\%) \\
p^{*}\end{array}$ & $\begin{array}{c}n(\%) \\
p^{*}\end{array}$ & $\begin{array}{l}n(\%) \\
p^{*}\end{array}$ & $\begin{array}{c}n(\%) \\
p^{*}\end{array}$ & $\begin{array}{c}n(\%) \\
p^{*}\end{array}$ & $\begin{array}{c}n(\%) \\
p^{*}\end{array}$ & $\begin{array}{l}\mathrm{n}(\%) \\
\mathrm{p}^{*}\end{array}$ & $\begin{array}{l}\mathrm{n}(\%) \\
\mathrm{p}^{*}\end{array}$ & $\begin{array}{l}n(\%) \\
p^{*}\end{array}$ & $\begin{array}{c}n(\%) \\
p^{*}\end{array}$ \\
\hline $\begin{array}{l}\text { A família acha } \\
\text { importante a AF }\end{array}$ & $77(65,3)$ - & $36(63,2)-$ & $\begin{array}{c}192(65,5) \\
0,053\end{array}$ & $\begin{array}{c}121(53,5) \\
0,864\end{array}$ & $\begin{array}{c}75(48,7) \\
0,331\end{array}$ & $\begin{array}{c}55(46,6) \\
0,352\end{array}$ & $22(45,8)-$ & $\begin{array}{c}23(39,7) \\
0,223\end{array}$ & $15(46,9)$ - & $\begin{array}{c}20(38,5) \\
0,213\end{array}$ \\
\hline $\begin{array}{l}\text { Pais têm tempo } \\
\text { para acompanhar }\end{array}$ & $\begin{array}{c}73(68,2) \\
0,129\end{array}$ & $\begin{array}{c}33(66,0) \\
0,118\end{array}$ & $\begin{array}{c}160(66,1) \\
0,672\end{array}$ & $\begin{array}{c}104(56,2) \\
0,155\end{array}$ & $\begin{array}{c}59(51,3) \\
0,562\end{array}$ & $\begin{array}{c}46(49,5) \\
0,158\end{array}$ & $\begin{array}{c}15(42,9) \\
0,497\end{array}$ & $\begin{array}{c}18(39,1) \\
0,496\end{array}$ & $\begin{array}{c}11(46,8) \\
0,598\end{array}$ & $\begin{array}{c}17(44,7) \\
0,292\end{array}$ \\
\hline $\begin{array}{l}\text { Os pais não ficam } \\
\text { constrangidos }\end{array}$ & $\begin{array}{l}6(60) \\
0,745\end{array}$ & $\begin{array}{c}2(50,0) \\
0,572\end{array}$ & $\begin{array}{l}12(48) \\
0,067\end{array}$ & $\begin{array}{c}20(83,3) \\
0,002\end{array}$ & $\begin{array}{c}4(33,3) \\
0,309\end{array}$ & $\begin{array}{c}4(40,0) \\
0,661\end{array}$ & $\begin{array}{l}1(20,0) \\
0,221\end{array}$ & $\begin{array}{c}6(75,0) \\
0,034\end{array}$ & $\begin{array}{c}1(100,0) \\
0,294\end{array}$ & $\begin{array}{c}1(25,0) \\
0,544\end{array}$ \\
\hline $\begin{array}{l}\text { Alguém de casa } \\
\text { pratica }\end{array}$ & $\begin{array}{c}53(73,6) \\
0,012\end{array}$ & $\begin{array}{c}24(75,0) \\
0,036\end{array}$ & $\begin{array}{c}133(68,6) \\
0,138\end{array}$ & $\begin{array}{c}81(55,1) \\
0,682\end{array}$ & $\begin{array}{c}44(51,8) \\
0,306\end{array}$ & $\begin{array}{c}36(47,4) \\
0,902\end{array}$ & $\begin{array}{c}15(46,9) \\
0,989\end{array}$ & $\begin{array}{c}14(42,4) \\
0,758\end{array}$ & $\begin{array}{c}6(40,0) \\
0,464\end{array}$ & $\begin{array}{c}10(37,0) \\
0,735\end{array}$ \\
\hline $\begin{array}{l}\text { Companhia de } \\
\text { parentes e amigos }\end{array}$ & $\begin{array}{c}45(80,4) \\
0,001\end{array}$ & $\begin{array}{c}25(80,6) \\
0,003\end{array}$ & $\begin{array}{c}64(65,3) \\
0,889\end{array}$ & $\begin{array}{c}48(57,8) \\
0,396\end{array}$ & $\begin{array}{c}18(42,9) \\
0,322\end{array}$ & $\begin{array}{c}22(44,9) \\
0,809\end{array}$ & $\begin{array}{c}3(25,0) \\
0,079\end{array}$ & $\begin{array}{c}4(20,0) \\
0,026\end{array}$ & $\begin{array}{c}4(40,0) \\
0,599\end{array}$ & $\begin{array}{c}6(46,2) \\
0,442\end{array}$ \\
\hline $\begin{array}{l}\text { Tem apoio das } \\
\text { pessoas próximas }\end{array}$ & $\begin{array}{c}59(64,8) \\
0,916\end{array}$ & $\begin{array}{c}31(66,0) \\
0,342\end{array}$ & $\begin{array}{c}156(65,3) \\
0,889\end{array}$ & $\begin{array}{c}105(56,1) \\
0,254\end{array}$ & $\begin{array}{c}54(48,6) \\
0,994\end{array}$ & $\begin{array}{c}42(44,7) \\
0,514\end{array}$ & $\begin{array}{c}14(42,4) \\
0,484\end{array}$ & $\begin{array}{c}15(36,6) \\
0,177\end{array}$ & $\begin{array}{c}12(48,0) \\
0,930\end{array}$ & $\begin{array}{c}11(37,9) \\
0,930\end{array}$ \\
\hline
\end{tabular}

*Teste Qui-quadrado.

\section{Discussão}

Como limitações do estudo identifica-se o baixo nível de escolaridade dos pais, o que pode gerar dificuldade em interpretar os dados principalmente nas respostas em relação a prática de $\mathrm{AF}$, as quais podem ser superestimadas. Da mesma forma, pode ter havido por parte dos respondentes uma confusão entre a prática de $\mathrm{AF}$ na escola e a AF no tempo livre.

Acredita-se que a principal barreira para a prática de $\mathrm{AF}$ dessas pessoas é a superproteção dos pais ${ }^{12}$. Com a falta de conhecimento os pais podem entender que 
TABELA 5 - Prática de AF pela percepção de cada aspecto ambiental como facilitador por sexo e faixa etária.

\begin{tabular}{|c|c|c|c|c|c|c|c|c|c|c|}
\hline \multirow{4}{*}{$\begin{array}{l}\text { Aspectos } \\
\text { Ambientais }\end{array}$} & \multicolumn{10}{|c|}{ Faixa etária } \\
\hline & \multicolumn{2}{|c|}{$07-10$ anos } & \multicolumn{2}{|c|}{ 11-19 anos } & \multicolumn{2}{|c|}{ 20-30 anos } & \multicolumn{2}{|c|}{$31-40$ anos } & \multicolumn{2}{|c|}{$>40$ anos } \\
\hline & Masc & Fem & Masc & Fem & Masc & Fem & Masc & Fem & Masc & Fem \\
\hline & $\begin{array}{l}n(\%) \\
p^{*}\end{array}$ & $\begin{array}{c}n(\%) \\
p^{*}\end{array}$ & $\begin{array}{c}n(\%) \\
p^{*}\end{array}$ & $\begin{array}{l}n(\%) \\
p^{*}\end{array}$ & $\begin{array}{l}n(\%) \\
p^{*}\end{array}$ & $\begin{array}{c}n(\%) \\
p^{*}\end{array}$ & $\begin{array}{c}n(\%) \\
p^{*}\end{array}$ & $\begin{array}{c}n(\%) \\
p^{*}\end{array}$ & $\begin{array}{l}n(\%) \\
p^{*}\end{array}$ & $\begin{array}{c}n(\%) \\
p^{*}\end{array}$ \\
\hline $\begin{array}{l}\text { Há locais próximos } \\
\text { de casa }\end{array}$ & $\begin{array}{c}48(71,6) \\
0,112\end{array}$ & $\begin{array}{c}21(65,6) \\
0,662\end{array}$ & $\begin{array}{c}113(68,1) \\
0,217\end{array}$ & $\begin{array}{c}76(61,3) \\
0,010\end{array}$ & $\begin{array}{c}30(49,2) \\
0,820\end{array}$ & $\begin{array}{c}26(46,4) \\
0,912\end{array}$ & $\begin{array}{c}10(50,0) \\
0,624\end{array}$ & $\begin{array}{c}11(44,0) \\
0,724\end{array}$ & $\begin{array}{c}9(56,3) \\
0,288\end{array}$ & $\begin{array}{c}7(28,0) \\
0,061\end{array}$ \\
\hline $\begin{array}{l}\text { Os locais são } \\
\text { acessíveis }\end{array}$ & $\begin{array}{c}50(70,4) \\
0,079\end{array}$ & $\begin{array}{c}22(68,8) \\
0,682\end{array}$ & $\begin{array}{c}111(63,8) \\
0,481\end{array}$ & $\begin{array}{c}75(56,4) \\
0,533\end{array}$ & $\begin{array}{c}36(54,5) \\
0,383\end{array}$ & $\begin{array}{c}30(50,0) \\
0,260\end{array}$ & $\begin{array}{c}11(55,0) \\
0,246\end{array}$ & $\begin{array}{l}9(39,1) \\
0,445\end{array}$ & $\begin{array}{c}8(53,3) \\
0,705\end{array}$ & $\begin{array}{c}5(19,2) \\
0,001\end{array}$ \\
\hline $\begin{array}{l}\text { A comunidade o } \\
\text { acolhe }\end{array}$ & $\begin{array}{c}59(66,3) \\
0,343\end{array}$ & $\begin{array}{c}28(65,1) \\
0,666\end{array}$ & $\begin{array}{c}152(64,1) \\
0,321\end{array}$ & $\begin{array}{c}100(51,3) \\
0,033\end{array}$ & $\begin{array}{c}56(41,7) \\
0,337\end{array}$ & $\begin{array}{c}46(44,2) \\
0,339\end{array}$ & $\begin{array}{c}16(43,2) \\
0,498\end{array}$ & $\begin{array}{c}18(38,3) \\
0,335\end{array}$ & $\begin{array}{c}10(45,5) \\
0,430\end{array}$ & $\begin{array}{c}6(18,2) \\
0,000\end{array}$ \\
\hline $\begin{array}{l}\text { Os profissionais são } \\
\text { preparados }\end{array}$ & $\begin{array}{c}55(64,7) \\
0,842\end{array}$ & $\begin{array}{c}29(65,9) \\
0,483\end{array}$ & $\begin{array}{c}147(63,4) \\
0,097\end{array}$ & $\begin{array}{c}78(47,6) \\
0,003\end{array}$ & $\begin{array}{c}57(48,3) \\
0,508\end{array}$ & $\begin{array}{c}40(44,0) \\
0,499\end{array}$ & $\begin{array}{c}13(40,6) \\
0,293\end{array}$ & $\begin{array}{c}19(39,6) \\
0,112\end{array}$ & $\begin{array}{c}11(45,8) \\
0,877\end{array}$ & $\begin{array}{c}12(31,6) \\
0,055\end{array}$ \\
\hline $\begin{array}{l}\text { Há projetos sociais } \\
\text { de AF }\end{array}$ & $\begin{array}{l}12(60) \\
0,588\end{array}$ & $\begin{array}{c}7(63,3) \\
1,000\end{array}$ & $\begin{array}{c}46(61,3) \\
0,462\end{array}$ & $\begin{array}{c}26(57,8) \\
0,544\end{array}$ & $\begin{array}{c}10(32,3) \\
0,036\end{array}$ & $\begin{array}{c}7(46,7) \\
0,896\end{array}$ & $\begin{array}{c}5(50,0) \\
0,876\end{array}$ & $\begin{array}{l}3(33,3) \\
0,655\end{array}$ & $\begin{array}{l}1(33,3) \\
0,713\end{array}$ & $\begin{array}{c}1(25,0) \\
0,533\end{array}$ \\
\hline $\begin{array}{l}\text { O clima favorece a } \\
\text { prática de AF }\end{array}$ & $\begin{array}{c}72(66,1) \\
0,358\end{array}$ & $\begin{array}{c}32(62,7) \\
0,891\end{array}$ & $\begin{array}{c}164(63,6) \\
0,229\end{array}$ & $\begin{array}{c}113(55,4) \\
0,629\end{array}$ & $\begin{array}{c}68(48,9) \\
0,939\end{array}$ & $\begin{array}{c}49(51,6) \\
0,216\end{array}$ & $\begin{array}{c}18(43,9) \\
0,516\end{array}$ & $\begin{array}{c}21(42,0) \\
0,966\end{array}$ & $\begin{array}{c}12(52,2) \\
0,119\end{array}$ & $\begin{array}{c}14(35,0) \\
0,595\end{array}$ \\
\hline $\begin{array}{l}\text { O ambiente é } \\
\text { seguro }\end{array}$ & $\begin{array}{c}63(63) \\
0,180\end{array}$ & $\begin{array}{c}31(66,0) \\
0,551\end{array}$ & $\begin{array}{c}164(65,3) \\
0,633\end{array}$ & $\begin{array}{c}108(54,0) \\
0,954\end{array}$ & $\begin{array}{c}66(49,6) \\
0,680\end{array}$ & $\begin{array}{c}49(46,7) \\
0,619\end{array}$ & $\begin{array}{c}15(40,5) \\
0,272\end{array}$ & $\begin{array}{c}15(34,1) \\
0,015\end{array}$ & $\begin{array}{c}12(48,0) \\
0,941\end{array}$ & $\begin{array}{c}14(33,3) \\
0,119\end{array}$ \\
\hline
\end{tabular}

*Teste Qui-quadrado.

o filho estará melhor cuidado se não se envolver em AF, logo a percepção deles que o filho sente-se capaz de praticar AF é um fator importante como facilitador para a prática e para possibilitar que a partir disto os outros itens do presente estudo sejam relevantes. Além disso, em um país em desenvolvimento como o Brasil, a força deste estudo está na população estudada e no número de indivíduos da amostra, onde a pesquisa possibilitou a participação de todos com DI maiores de 06 anos, diferentemente dos estudos realizados em países desenvolvidos que possuem uma realidade diferente ${ }^{17}$.

Os resultados sobre a prática de AF deste estudo são preocupantes, pois relevam um baixo envolvimento das pessoas com $\mathrm{DI}$ em $\mathrm{AF}$, apesar dos benefícios à saúde que poderiam alcançar sendo fisicamente mais ativos ${ }^{17-18}$. Há evidências de que $\mathrm{AF}$ reduz mortalidade e morbidade por doença cardiovascular, hipertensão, obesidade, osteoporose e doença mental. Destaca-se que essas doenças são elevadas em pessoas com DI ${ }^{18}$ e que essa população experimenta mais prejuízos para a saúde que a população geral ${ }^{19}$. Os benefícios para a saúde de ser fisicamente ativo tem sido alvo de algumas pesquisas, entretanto, os profissionais envolvidos na intervenção comunitária não têm conseguido influenciar as pessoas de forma eficaz na aderência aos programas de AF para a população em geral ${ }^{20}$.

Sobre os aspectos pessoais, o estudo revelou que principalmente os homens que apresentam os facilitadores de gostar de AF e sentir-se capaz de praticar, são os que mais o fazem no tempo livre, bem como as mulheres a partir dos 20 anos. Pela percepção dos responsáveis esses itens são facilitadores da prática em todas as idades. Resultados parecidos foram encontrados em estudos que apontam que os facilitadores incluíram a preferências das pessoas em praticar AF, o desejo de ser ativo, ter habilidades para praticar ${ }^{18,19}$. Sendo assim, devido à limitação intelectual que as pessoas com DI possuem em dicernir informações e tomar decisões, cabe às pessoas em volta fazer o estímulo para que a elas gostem de ser ativas e descubram juntas qual a atividade mais prazerosa e que melhor se adpte às suas condições. Partindo dos princípios da inclusão, a ideia atual para a relação com as pessoas com deficiência centra-se em não perceber a pessoa em razão da sua deficiência, de 
suas limitações, mas procurar estimulá-la para que possa desenvolver-se na medida de suas capacidades, no ambiente em que vive ${ }^{12}$.

A timidez não foi apontada como barreira para a prática, no entanto, o fato de não ser tímido estava associado com a prática em meninas e em senhoras, como também em homens de 31 a 40 anos. Autores também encontraram que o fato de não se sentir aceito é percebido como uma barreira para a participação em atividades de lazer ${ }^{22}$. Provavelmente essa condição tem relação com os estímulos que a pessoa com DI recebe e que melhorem sua autoestima no momento em que ela tem autonomia, independência e se sente feliz junto aos demais, sendo aceita em seu ambiente.

Dentre os aspectos caracterizados como ambientais, o mais destacado como barreira foi o fato de não haver projetos sociais próximos de casa. Esse resultado corrobora com outros estudos que mostram que as questões financeiras, falta de opções, a falta de apoio social e de apoio político e financeiro, instalações acessíveis, a proximidade da localização, boas oportunidades para a prática, pessoas especializadas, interferem e são preditores de baixo nível de AF para pessoas com $\mathrm{DI}^{21-22}$. Um estudo também mostrou que a questão mais crítica para o baixo nível de AF foi a falta de políticas claras. Ainda acrescentam que, na lógica do desenvolvimento de uma política, seria necessária a formação e o apoio do pessoal envolvido ${ }^{23}$.

Os demais itens foram descritos como facilitadores, no entanto, ao ser verificada a associação entre eles e a prática de AF poucos mostraram resultados significativos consideravelmente, sendo eles: a comunidade o acolher de forma adequada; os profissionais serem preparados para atendê-los e haver locais disponíveis próximos de casa. Percebe-se que com a existência de projetos sociais com oferta de atividades físicas para o tempo livre, esses itens seriam contemplados. Em um estudo afirmaram que o apoio social está associado a maiores taxas de participação em atividades de lazer e concluíram que, mesmo que a família facilite a participação em atividades de lazer, não necessariamente aumenta a participação autônoma e independente ${ }^{24}$. Outro estudo mostrou que as barreiras ambientais influenciam mais do que as pessoais na prática de atividades no lazer que incluíam $\mathrm{AFs}^{25}$, o que reforça a necessidade de investimento nesse aspecto.

Quanto a percepção dos pais sobre a influência dos aspectos sociais, a falta de companhia para praticar foi o principal fator descrito como barreira e está associado com a inatividade física no tempo livre. A companhia de outras pessoas aumenta o interesse na prática de $\mathrm{AFs}^{26}$. O apoio social, mesmo parecendo barreira comum para todos, para pessoas com DI muitas vezes assume uma forma diferente. Pode requerer ajuda concreta para facilitar a participação em AF, tais como planejamento e transporte, e não simplesmente o incentivo ou companheirismo. Para aqueles adultos com DI que não têm capacidade de leitura, o apoio social pode ser necessário até mesmo para tomar conhecimento das opções disponíveis para $\mathrm{AF}$, pois podem não ter consciência das opções que existem ${ }^{22}$.

Assim, enquanto apoio e encorajamento de outros ajuda a reforçar a participação em AF na população em geral, parece ser um fator essencial para adultos com DI serem capazes de participar em AF. Além disso, a falta de incentivo pode ser uma barreira para ambas populações, mas o desânimo dos outros que estão ao redor é uma barreira percebida exclusivamente para adultos com DI. O próprio controle sobre as escolhas das atividades não está sempre disponível para as pessoas com DI, mas, sim, de seus responsáveis.

Já o fato dos pais não se sentirem constrangidos com os filhos praticando AF foi apontado como facilitador da prática com associação significativa. $\mathrm{O}$ apoio emocional da família, dos amigos e profissionais foi apontado como facilitador 
de uma maior participação em atividades de lazer das pessoas com deficiência ${ }^{27-29}$.

A sociedade tem que promover uma abordagem inclusiva para programas comunitários e serviços que reconheçam e apoiem as necessidades de pessoas com deficiência ${ }^{23-30}$. Embora normalmente haja uma necessidade de serviços de AF separados ou adaptados, o fato de que esses programas são pouco oferecidos na maioria das comunidades em todo o mundo, especialmente nos países mais pobres, onde os recursos dedicados ao cuidado e bem-estar são limitados, há a necessidade de maior número de programas inclusivos de $\mathrm{AF}$ que forneçam elementos de adaptação para as pessoas com deficiência ${ }^{8}$.

O fato de sentir-se capaz de praticar atividade foi o mais associado com a prática de AF no tempo livre. Possuir companhia para a prática está associado com a prática de $\mathrm{AF}$ principalmente nos mais novos e os profissionais serem preparados para promover a prática de AF das pessoas com DI também é um facilitador. As principais barreiras para a prática de $\mathrm{AF}$ das pessoas com $\mathrm{DI}$, percebidas pelos seus responsáveis, foram a falta de dinheiro, falta de projetos sociais, preferir outra atividade ao invés de $\mathrm{AF}$ e falta de companhia para praticar.

Conclui-se que as barreiras e facilitadores estão normalmente ligados ao estímulo que as pessoas com DI recebem. As barreiras mais frequentes para prática de AF foram falta de dinheiro e de projetos sociais perto de casa voltados a pratica de $\mathrm{AF}$, visto que grande parte da amostra do estudo é de classe social baixa. Com relação aos facilitadores, observou-se que tempo para praticar AF, ser capaz de praticar $\mathrm{AF}$ e o entendimento que a família tem sobre a importância da pratica de AF são aspectos primordiais. Ter companhia para prática de AF foi relatado como como um facilitador associado com a prática de AF.

Percebe-se com o estudo que há um entendimento adequado sobre a importância da AF no tempo livre na vida das pessoas com DI, entretanto, é necessário que sejam desenvolvidas políticas públicas e projetos sociais, que permitam o acesso dessas pessoas a prática de AF. Essas oportunidades de práticas de AF irão refletir num estilo de vida ativo e numa consequente melhor qualidade de vida.

\section{Agradecimentos}

Agradecemos a Federação das Associações de Pais e Amigos dos Excepcionais do Rio Grande do Sul (FEAPAES-RS) pela viabilização e apoio para realização do estudo.

\section{Contribuição dos autores}

L. G. Jung (0000-0003-2505-1368) Contribuições: elaboração do projeto, análise e interpretação dos dados, redação do artigo e aprovação final da versão a ser publicada. A. X. Kalinoski (0000-0003-2594-2531) Contribuições: elaboração do projeto, análise e interpretação dos dados, redação do artigo e aprovação final da versão a ser publicada. A. C. Marques (0000-0002-1866-4169) Contribuições: orientador do estudo, participou da elaboração do projeto, análise e interpretação dos dados, redação do artigo e aprovação final da versão a ser publicada.

\section{Referências}

1. Instituto Brasileiro de Geografia e Estatística(IBGE). Tábua completa de mortalidade para o Brasil: Breve análise da evolução da mortalidade no Brasil. Brasília, 2015. Disponível em: ftp://ftp.ibge.gov.br/Tabuas_Completas_de_Mortalidade/Tabuas_Completas_de_ Mortalidade_2015/tabua_de_mortalidade_analise.pdf. Acesso em 20 de abril de 2017.

2. Pitanga FJG. Epidemiologia, atividade física e saúde. Rev Bras Ciên e Mov. 2002;10(3):49-54. 
3. Bauman AE. Updating the evidence that physical activity is good for health: an epidemiological review 2000-2003. J Sci Med Sport. 2004;7(1):6-19.

4. Lee IM, Shiroma EJ, Lobelo F, Puska P, Blair SN, Katzmarzyk PT. Effect of physical inactivity on major non-communicable diseases worldwide: an analysis of burden of disease and life expectancy. Lancet. 2012;380(9838):219-29.

5. Cooper S-A, Morrison J, Melville C, Finlayson J, Allan L, Martin RG, et al. Improving the health of people with intellectual disabilities: Outcomes of a health screening programme after 1 year. J Intellect Disabil Res. 2006;50(Pt 9):667-77.

6. Carmeli E, Barak S, Morad M, Kodesh E. Physical exercises can reduce anxiety and improve quality of life among adults with intellectual disability. Int SportMed J. 2009;10(2):77-85.

7. Finlayson J, Jackson A, Cooper SA, Morrison J, MelVille C, Smiley E, et al. Understanding Predictors of Low Physical Activity in Adults with Intellectual Disabilities. J Appl Res Intellect Disabil. 2009;22(3):236-47.

8. Rimmer JH, Marques AC. Physical activity for people with disabilities. Lancet. 2012; 380(9838):193-5.

9. Hilgenkamp T, Reis D, Wijck RV, Evenhuis HM. Physical activity levels in older adults with intellectual disabilities are extremely low. Res Dev Disabil. 2012;33(2):477-83.

10. Malta DC, Castro AM, Cruz DkA, Gosh CS. A Promoção da saúde e da atividade física no Sistema Único de Saúde. Rev Bras Ativ Fis e Saúde 2008;13(1):24-27.

11. Bauman AE, Reis RS, Sallis JF, Wells JC, Loos RJF, Martin BW. Correlates of physical activity: why are some people physically active and others not? Lancet. 2012;380(9838):258-71.

12. Marques AC. O perfil do estilo de vida de pessoas com Síndrome de Down e normas para avaliação da aptidão física. [tese de doutorado] Porto Alegre: Universidade Federal do Rio Grande do Sul; 2008.

13. Sousa TF, Santos SFS, José HPM. Barreiras percebidas à prática de atividade física no Nordeste do Brasil. Pensar a Prática. 2010;13(1):1-15

14. Seron BB, Arruda GA, Greguo M. Facilitadores e barreiras percebidas para a prática de atividade física por pessoas com deficiência motora. Rev Bras Ciênc Esporte. 2015;37(3):214-21.

15. Jung LG. Atividades diárias e percepção de Barreiras e Facilitadores para Prática de Atividade Física de Pessoas com Déficit Intelectual. [dissertação de mestrado]. Pelotas: Universidade Federal de Pelotas; 2013.

16. World Health Organization - WHO. Recomendações de Atividade Física [online]. Disponível em: url: http://www.who.int/dietphysicalactivity/publications/ recommendations/en/

17. Rimmer JH, Riley B, Wang E, Rauworth A, Jurkowski RJ. Physical activity participation among persons with disabilities: barriers and facilitators. Am J Prev Med. 2004;26(5):419-25

18. Lennox N, Beange H, Parmenter T, Santos-Teachout R, Evenhuis H, Kerr M, et al. Health Guidelines for Adults with an Intellectual Disability, 2002 [online]. Disponível em: url: http://cddh.monash.org/assets/health-guidelines-for-adults-id.pdf

19. Valk VSL. Healthy persons with intellectual disabilities in an inclusive society. J Policy Pract Intellect Disabil. 2009;6(2):77-80.

20. Marques, AC. Perfil da qualidade de vida de pessoas com Síndrome de Down maiores de 40 anos em Santa Catarina. [dissertação de mestrado]. Florianópolis: Universidade Federal de Santa Catarina; 2000.

21. Schijndel-Speet MV, Evenhuis HM, Wijck RV, Empelen PV, Echteld MA. Facilitators and barriers to physical activity as perceived by older adults with intellectual disability. Intellect Dev Disabil. 2014;52(3):175-86.

22. Bodde AE, Seo DC. A review of social and environmental barriers to physical activity for adults with intellectual disabilities. Disabil Health J. 2009;2(2):57-66.

23. Temple VT, Walkley JW. Perspectives of constraining and enabling factors for healthpromoting physical activity by adults with intellectual disability. J Intellect Dev Disabil. 2007; 32(1):28-38.

24. Abraham C, Gregory N, Wolf L, Pemberton R. Self esteem, stigma and community participation amongst people with learning difficulties living in the community. $J$ Community Appl Soc Psychol. 2002;12(6):430-43.

25. Badia M, Orgaz B, Verdugo MA, Ullán AM, Martínez MM. Personal factors and perceived barriers to participation in leisure activities for young and adults with developmental disabilities. Res Dev Disabil. 2011;32(6):2055-63. 
26. Stanish HI, Curtin C, Must A, Phillips S, Maslin M, Bandini LG. Physical Activity Enjoyment, Perceived Barriers, and Beliefs Among Adolescents With and Without Intellectual Disabilities. J Phys Act Health. 2016; 13(1):2-10.

27. Lin JD, Lin PY, Lin LP, Changa YY, Wu SR, Wu JL. Physical activity and its determinants among adolescents with intellectual disabilities. Res Dev Disabil. 2010;31(1):263-69.

28. Njelesani J, Leckie K, Drummond J, Cameron D. Parental perceptions of barriers to physical activity in children with developmental disabilities living in Trinidad and Tobago. Disability Rehabilitation. 2015;37(4):290-5.

29. Taliaferro AR, Hammond L."I Don't Have Time”: Barriers and Facilitators to Physical Activity for Adults With Intellectual Disabilities. Adapt Phys Activ Q. 2016;33(2):113-33.

30. Shields N, Synnot AJ. Perceived barriers and facilitators to participation in physical activity for children with disability: a qualitative study. BMC Pediatr. 2016;16(1):9.

Endereço para

Correspondência

Laura Garcia Jung

lauragjung@gmail.com
Rua Olegário Mariano, 231 / casa 480

CEP: $96065-390$

Telefone: 053 98155-3005
Recebido

Revisado

$1 / 02 / 2017$

$01 / 05 / 2017$

$18 / 05 / 2017$

$18 / 05 / 2017$ 